\title{
The Recombination in a FRW Universe with a Variable Cosmological Term
}

\author{
N. Pires and J. A. S. Lima \\ Departamento de Física - UFRN, CP 1641, 59072-970 Natal, RN - \\ Brasil
}

\begin{abstract}
The effects of a time-dependent $\Lambda$ term in the recombination epoch are analysed taking into account the main physical processes occurring in the primordial plasma. For a vacuum decaying into photons, we show that the recombination begins when the Universe was smaller and denser, leading to a greater recombination rate. These results may have several implications to the large scale structure. In particular, the earlier recombination means that more time is available to the evolution of density perturbations.
\end{abstract}

\section{The Model}

We consider an adiabatic model with a $\Lambda$-term decaying into photons (Lima 1996 and Lima and Trodden 1996), given by $c^{2} \Lambda_{v}(t)=8 \pi G \beta \rho_{T}$, where $\rho_{T}$ is the total energy density (radiation, matter and vacuum). The parameter $\beta<0.16$ is constrained from nucleosynthesis studies (Maia et al. 2000). In this model the temperature law scales as $T_{\gamma} \propto a^{1-\beta}$, where $a$ is the scale factor. For FRW models with a $\Lambda$-term, the age of the Universe reads

$$
H_{0} t_{0}=\int_{0}^{1}\left(\frac{(1-\beta) x^{(1-3 \beta)}}{\Omega_{m 0}+\left(1-\beta-\Omega_{m 0}-\lambda_{0}\right) x^{(1-3 \beta)}+\lambda_{0} x^{3(1-\beta)}}\right)^{1 / 2} d x
$$

where $H_{0}=100 h \mathrm{Mpc}^{-1} \mathrm{~km} \mathrm{~s}^{-1}$ is the Hubble constant, $\lambda_{0}=\Lambda_{c} / 3 H_{0}^{2}$ is the cosmological constant and $\Omega_{m 0}=\left(8 \pi G / 3 H_{0}^{2}\right) \rho_{m 0}$ is the matter density parameter. The index " 0 " denotes the present day values. Assuming that the Universe is a mixture of protons, electrons and hydrogen, we consider a variety of physical processes envolving matter and radiation during and after recombination: photon drag, ionization due to radiation and electronic collisions, recombination cooling and Compton heating-cooling (Opher et al. 1998).

The results have been obtained using a hydrodynamical code (including the above processes), and are summarized in the table below. All models are parametrized by $\Omega_{m 0}, h, \beta$, and $\lambda_{0}$. This table yields the temperature of the radiation, $T_{\gamma r e c}$ and the redshift, $z_{r e c}$, at the recombination epoch, which is assumed to occur when $50 \%$ of the electrons have been captured. The last column of this table yields the age of the Universe as given by (1). 


\begin{tabular}{|cccccccc|}
\hline \hline Model & $\Omega_{m 0}$ & $h$ & $\beta$ & $\lambda_{0}$ & $T_{\gamma r e c}(K)$ & $z_{\text {rec }}$ & Age $($ Gyr $)$ \\
\hline \hline A1 & 0,024 & 0,73 & 0,1 & 0,0 & 3900 & 3201 & 13,1 \\
A2 & $"$ & $"$ & 0,05 & 0,0 & 3740 & 2003 & 13,0 \\
A3 & $"$ & $"$ & 0,0 & 0,876 & 3540 & 1296 & 20,3 \\
A4 & $"$ & $"$ & 0,1 & 0,876 & 3920 & 3220 & 25,1 \\
A5 & $"$ & $"$ & 0,0 & 0,0 & 3540 & 1296 & 12,9 \\
\hline B1 & 0,05 & 0,5 & 0,1 & 0,0 & 3900 & 3201 & 18,8 \\
B2 & $"$ & $"$ & 0,05 & 0,0 & 3740 & 2003 & 18,6 \\
B3 & $"$ & $"$ & 0,0 & 0,85 & 3540 & 1296 & 26,7 \\
B4 & $"$ & $"$ & 0,1 & 0,85 & 3920 & 3220 & 31,7 \\
B5 & $"$ & $"$ & 0,0 & 0,0 & 3540 & 1296 & 18,3 \\
\hline C1 & 0,1 & 0,73 & 0,1 & 0,0 & 4070 & 3357 & 12,5 \\
C2 & $"$ & $"$ & 0,05 & 0,0 & 3900 & 2093 & 12,3 \\
C3 & $"$ & $"$ & 0,0 & 0,8 & 3720 & 1362 & 16,1 \\
C4 & $"$ & $"$ & 0,1 & 0,8 & 4070 & 3357 & 18,6 \\
C5 & $"$ & $"$ & 0,0 & 0,0 & 3720 & 1362 & 12,0 \\
\hline D1 & 0,1 & 0,5 & 0,1 & 0,0 & 3990 & 3283 & 18,3 \\
D2 & $"$ & $"$ & 0,05 & 0,0 & 3820 & 2048 & 17,9 \\
D3 & $"$ & $"$ & 0,0 & 0,8 & 3620 & 1325 & 23,5 \\
D4 & $"$ & $"$ & 0,1 & 0,8 & 3990 & 3283 & 27,1 \\
D5 & $"$ & $"$ & 0,0 & 0,0 & 3620 & 1325 & 17,6 \\
\hline
\end{tabular}

\section{CONCLUSIONS}

For models with cosmological constant (or standard CDM), same results already established are also diplayed: The recombination begins in a higher redshift if the Universe is denser. The Universe is older if the cosmological constant increases (for the same $h$ ). For models with a decaying vacuum into photons the main results are:

1) $\beta \neq 0$ always increases the age of the Universe.

2) The recombination temperature $\left(T_{\gamma r e c}\right)$ is greater and the same happens with the redshift $\left(z_{r e c}\right)$.

3) The $\beta$ parameter has also a deep influence on the late time evolution of fluctuations. In particular, comparing several models at equal redshift, the Jeans mass is smaller if the $\beta$ parameter increases.

\section{References}

Lima J. A. S. 1996, Phy. Rev. D., 54, 2571

Lima J. A. S. \& Trodden M. 1996, Phy. Rev. D., 53, 4280

Maia J., Lima J. A. S. \& Pires N., Constraints from Big Bang Nucleosynthesis on a Time Varying Cosmological "Constant", in this book.

Opher R., Pires N. \& de Araujo J.C.N. 1997, MNRAS, 285, 811

Pires N. 1999, PhD Thesis, IAG-USP 